\title{
Volumetric Image Guidance via a Stereotactic Endoscope
}

\author{
Ramin Shahidii ${ }^{1}$, Bai Wang ${ }^{1}$, Marc Epitaux ${ }^{1}$, Robert Grzeszczuk ${ }^{2}$, John Adler ${ }^{1}$ \\ ${ }^{1}$ Stanford University, Department of Neurosurgery, Image Guidance Laboratory, \\ 300 Pasteur Dr., MC: 5327, Stanford, California 94305-5327, USA \\ \{shahidi, bai, mepitaux, adler\}@igl.stanford.edu \\ http://igl.stanford.edu \\ ${ }^{2}$ Silicon Graphics, Inc., Mountain View, California 94943, USA \\ rg@sgi.com
}

\begin{abstract}
We have developed a surgical setup based on modern frameless stereotactic techniques that enables surgeons to visualize the field of view of the surgical endoscope, overlaid with the real-time and volumetrically reconstructed medical images, of a localized area of the patient's anatomy. Using this navigation system, the surgeon visualizes the surgical site via the surgical endoscope, while exploring the inner layers of the patient's anatomy by utilizing the three-dimensionally reconstructed image updates obtained by pre-operative images, such as Magnetic Resonance and/or Computed Tomography Imaging. This system also allows the surgeon to virtually "fly through and around" the site of the surgery to visualize several alternatives and qualitatively determine the best surgical approach. Moving endoscopes are tracked with infra-red stereovision cameras and diodes, allowing the determination of their spatial relation to the target lesion and the fiducial based patient/image registration.
\end{abstract}

\section{Introduction}

Recent developments in computation technology have fundamentally enhanced the role of medical imaging, from diagnosis to computer-aided surgery. Today, Computer assisted methods provide on-the-fly information for dynamic navigation, analysis and inspection of the 3-D image structures during surgical procedures. Moreover, conventional stereotactic techniques, in which the frame of reference is bolted to the skull, are being replaced by frameless systems that incorporate fiducial markers for registration. The combination of these two methods provide the surgeon with interactive and intuitive access to the imaging data, like Magnetic Resonance Imaging (MRI), Computed Tomography (CT) in an ongoing surgical procedure.

The objective of our paper is to describe a system for a real-time 3-D surgical navigation using an operating endoscope. This system allows for the overlay of volumetrically reconstructed patient images onto the view of the surgical field as seen through the endoscope. This technique permits surgeons to navigate around the patient to explore the inner anatomical structures of the patient, thus providing "onthe-fly" volumetric and 2-D information for planning and navigational purposes. The important part of this system is the navigation software, which does not burden the surgeon with the implementation details of the computation model. The graphic interface provides surgeons with an interactive environment, to directly address the problems associated with the task by selecting from a variety of possible visualization capabilities. All other aspects, such as object tracking, are carried out automatically. 


\section{Background}

In the past, the task of correlating preoperative and intraoperative imaging studies was left to the surgeon and depended on his/her knowledge of human anatomy. Stereotaxi was one of the first procedures to effectively corollate preoperative images with the patient's physical anatomy during the operation.

\subsection{Frame Based Stereotaxi}

Up to recent years, the most common use of stereotaxi was for biopsies, for which a surgical trajectory device and a frame of reference were used [1]. Traditional framebased methods of stereotaxy defined the intracranial anatomy with reference to a set of fiducial markers, attached to a stereotactic frame [2]. The stereotactic frame-bolted into the patient's skull prior to imaging and worn throughout the duration of the procedure-was used for registering the patient with the pre-operative data [3]. The trajectories prescribed by trigonometric calculations obtained from pre-operative images were interpolated into the operative field using a trajectory enforcement device. This device, placed on top of the frame of reference was used to guide the biopsy tool to the target lesion based on prior calculations [4]. Use of a mechanical frame allowed for high localization accuracy, but caused patient discomfort and limited surgical flexibility. In addition, such surgical techniques did not allow the surgeon to visualize the approach of the biopsy tool to the lesion.

\subsection{Two-Dimensional Frameless Image Guided Stereotaxi}

There has been an emergence of image-guided techniques for registration that are eliminating the need for the frame all together. The first frameless stereotactic system utilized an articulated robotic arm to register pre-operative imaging with the patient's anatomy in the operating room $[5,6,7]$. The next step was the use of sonic devices for tracking the instruments in the operating room [8]. A significant evolution of the frameless system was the introduction of Optical Tracking System (OTS) which utilized infra-red diodes and vision-cameras to track a moving object in the operating room $[9,10,11,12]$. All three systems mentioned above, use externally placed markers on the patient to register pre-operative imaging with the patient's anatomy in the operating room [13]. These frameless methods, however, have limited positional accuracy and use limited image data displays as 2-D views. In addition, they tend to lose their spatial registration with respect to pre-operative data [14]. For example, in neurosurgical applications, the deformation of the brain due to CSF drainage (brainshift) causes significant errors in the localization of the targeted lesion.

\subsection{Three-Dimensional Frameless Image Guided Stereotaxi}

In 1994, several groups introduced the concept of intraoperative volumetric image guidance, using various approaches $[15,16,17,18]$. Volumetric imaging techniques greatly enhance a surgeon's ability to create a plan prior to surgery, to follow it during surgery, and to modify the surgical approach based on intra-operative information. The intraoperative use of 3-D imaging has been limited, due to a lack of computation power. However, recent advances in graphic hardware designs, provides the means for effective utilization of such 3-D images for intraoperative guidance, such as the fusion of preoperative $3-\mathrm{D}$ images with the video images of the surgical site $[19,20,21]$. 


\section{Overview}

The main draw backs of fiberoptic endoscopy are that, it can only display visible surfaces, and can not be used inside an opaque tissue. Virtual endoscopy on the other hand, can make an opaque tissue transparent and look beyond the visible surface. Thus, the correlation of the actual endoscopic video images with the volumetrically reconstructed imaging data, provides precise additional exposure of the pathology and its surrounding tissue during the surgery. A hypothetical scenario of our proposed system's use in the operating room will help to delineate its features.

Prior to obtaining pre-operative images, six markers (fiducials) are placed on the patient. These are small $(\sim 1 \mathrm{~mm})$, stick-on markers surrounded by pantopaque-filled spheres glued to the skin. Pantopaque is an oil-based, iodine containing X-ray contrast agent that until recently was used for myelograms. The iodine makes the fiducials visible on CT images while the oil base renders it visible on MRI examinations. The image data will be transferred to the SGI Infinite Reality workstation (Silicon Graphics Inc., Mountain View, CA), via the hospital's computer network. The volume will be rendered, and (in the case of multiple imaging modalities) fused. If necessary, the image data will then be segmented to allow detailed visualization in the appropriate anatomic context of the lesion, the vascular structures, and the fiducials. Segmentation of the fiducial markers, the brain, the vascular system (from CTA or MRA) and the surface of the brain will be fully automatic. The segmentation of the lesion (if applicable), however, would only be partly automatic, since the irregular anatomy surrounding lesions are currently too unpredictable for automatic segmentation.

In the operating room, the patient will be positioned in the usual fashion and the registration process will be accomplished using the Optical Tracking System (Image Guided Technologies Inc., Boulder, CO). Optical tracking is a technique that allows a unique detection of flashing objects with known frequencies in 3-D space. Diode markers can also be added to conventional surgical tools (e.g., probes or endoscopes) to enable their tracking in space. Using such tracking techniques, the position of the encoded surgical pointer in 3-D space is automatically recognized. By pointing the encoded surgical probe to the actual markers and moving the computer cursor on the markers' imaging counterparts, registration of the volumetric image and the patient's actual physical anatomy can be accomplished. At this point, since the patient and the 3-D image are registered and the surgical probe can be tracked with respect to the patient, the virtual counterpart of the probe can also be tracked with respect to the 3-D image. A line extending from the displayed image of the probe indicates the trajectory of the planned approach.

Moving the tool, automatically leads to a change in the displayed potential trajectory. This system simplifies the planning of a minimally invasive approach to a direct and interactive task. In addition to displaying the probe's trajectory in 3-D, the location of the infra-red diodes on the probe will determine the precise location and depth of the tool, hence, the precise location and depth of the operative site. 
The surgical probe can also be replaced with the diode encoded rigid fiberoptic endoscope (PS Medical, Goleta, CA). The volumetric images of the surgical corridor (focused on the surgical site) are then re-oriented to show the endoscope's virtual birds-eye-view. This information allows the workstation to automatically display the real time 3-D images of the surgical corridor in context, as oriented to the surgical approach. 3-D reformatting uses volume display techniques and allows instantaneous variations of transparency. With this technique, deep as well as superficial structures can be seen in context, considerably enhancing intra-operative guidance. These 3-D virtual endoscopic views can be seen next to the endoscope's actual views.

\section{Methods}

To incorporate this system, the following steps have been taken: 1) deployment of a fast 3-D Engine for a reconstruction of the medical data, 2) development of various visualization and navigation tools in a user friendly environment, 3) 3-D patient/ image registration and real-time tracking of the surgical tools, and 4) perspective rendering of the localized 3-D data to match the fiberoptic endoscope images.

\subsection{Fast Volume Rendering Engine}

Direct volume rendering is a technique for displaying sampled scalar or vector fields without fitting any geometric primitives to the data $[32,33]$. Instead, the volume is sampled directly with a set of rays emanating from the eye, hence the name of the technique: "Ray Tracing" [34,35]. As each ray is traversed, the optical properties along the path are computed. These may include absorption, reflection, refraction, and emission. In addition, it is possible to devise modes that do not have a natural physical interpretation, but which can provide useful information, nevertheless.

In conventional implementations, each ray is traced from the eye to its point of termination before the next ray is considered. However, many newer algorithms $[36,37]$ choose to process the rays simultaneously taking advantage of spatial coherence and the locality of the reference, to dramatically improve performance. This approach is particularly attractive on platforms capable of hardware texturemapping. Drawing a texture-mapped plane perpendicular to the line of sight produces identical results to sampling a volume with a set of rays under orthographic projection. Similarly, ray casting under perspective projection can be accomplished by drawing a set of texture-mapped concentric spheres centered at the eye (Fig. 1).

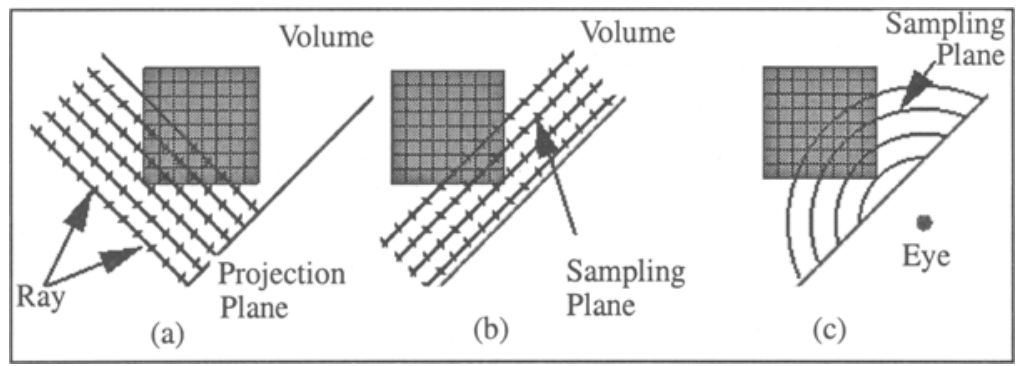

Figure 1: Ray-casting with orthographic projection. a) ray order, b) sampling surface and,

c) sampling with perspective projection. 
The ray casting method we adopted employs a general framework which unifies the treatment of volumes and geometry. A volume is characterized by both appearance and geometry. The volume's appearance is a collection of voxels, while the geometry is given by a set of 3-D Simplices (Tetrahedra), which define the region of interest. The volume is rendered by polygonizing the geometry into a series of triangles that are texture-mapped with appearance data. This results in a highly flexible approach, allowing us to take full advantage of available graphics hardware. In addition, the technique is flexible enough to allow for many desirable operations such as selecting arbitrarily shaped regions of interest, multi-modality rendering, and rendering both volumetric and surface rendering as embedded geometrical objects.

It is critical that the surgeons be able to interact with the system in a manner that does not hinder their performance. Therefore, the visuals needed for navigation have to be generated at highly interactive rates, which is a hard task to achieve using conventional, software-only solutions. As a result, we have decided to take advantage of the graphic board of SGI-IR Workstation, which conducts many graphical calculations in the hardware, thus substantially increasing the rendering process. Our software is deployed on top of C++, OpenGL, SGI's Inventor and VI Tool-Kits. The speed of our volume rendering varies from 15 frames/sec to 45 frames/sec depending on the data characteristics (between 20MB to 60MB) on a SGI-IR 4xproc.

\subsection{Visualization and Navigation Software (Genesis2000)}

In addition to the 3-D Engine, a 2-D Engine is implemented in our volumetric navigation software. The 2-D Engine provides the user with orthogonal views (Axial, Sagital, Coronal). The implemented 2-D Engine, however, is capable of displaying the orthogonal view in 3-D as well. By applying six cutting planes to the 3-D Engine, three orthogonal views for axial, Sagital, and Coronal views can be presented. When the 2-D Engine is on slice mode, both cutting planes are enabled. When the 2-D Engine is on non-slice mode, only one cutting plane is enabled, and will cut out everything between the viewer and the plane. When necessary, the 2-D Engine supports a reverse view, by flipping the effective cutting plane area and reversing the view direction. The 2-D Engine can also display non-orthogonal slices (oblique slices). When in slice mode, due to the small gap between the two cutting planes, performance is in real-time. For a $512 \times 512 \times 128$ data set, three $512 \times 5122$-D views and 32 bit color images can be generated at about 30 frames/second on the SGI-IR.

We have implement various tool boxes to our software package. The main tool boxes are the "Loader" (loading and saving the medical data-sets), the "Basic Tool Box" (histogram, opacity, color, and light adjustments), the "Basic Segmentation Tool Box" (seed-fill, semi-automated contouring, and basic analysis), the "Basic Registration Tool Box" (multimodality and patient/image fusion), and the "Basic Navigation Tools" (viewing, registration and navigation parameters). These tool boxes are used to control the current system's lookup table, in order to interactively update the images that are being modified. The system lookup table is $32 \mathrm{bit}$, i.e., for red, green, blue and alpha. The tool-boxes control every channel of the lookup table separately, so different tissues can be assigned different visualization properties. 
The user interface is probably the most crucial part of our system. It is fully conformed towards our surgeon's requirements and was designed taking into consideration their constant feed back. The interface is designed to be intuitive and easy to use. It does not burden the physician with the details of implementing the computational model. All aspects of graphics and analysis are carried out automatically. It also remembers the windowing arrangements for all its users, and it has the ability to save the rendering settings to minimize the initialization time. In order to maintain the simplicity of the interface without losing its functionality, our interface is specifically designed for surgical navigation purposes.

\subsection{Registration and Tracking Surgical Instrument}

The basic principle involved in tracking the surgical instruments, is triangulation which is used for the recovery of the depth of an indicator from vision-cameras. In our case, the indicators are the infra-red diodes that are being tracked by three infra-red cameras in the surgical space. Using the computer-vision technique, the indicator must be established between features from the three images (from infra-red cameras) that correspond to some physical property in space (infra-red signal). Then, provided that the relative positions of the centers of projection, the effective focal length, the relative orientation of the optical axis, and the sampling interval of each camera are all known, the closest distance of the object to the camera can be calculated $[28,29]$.

The surgical endoscope and other surgical tools must work in synchronisity in order to maintain a dynamic model of the surgical site. Our main challenge was to keep all sensors in a sub-millimeter spatial registration, and maintain a detailed dynamic model of the motion. We had build a special interface so that two or three objects could be tracked at the same time. In addition, we have built an infra-red-based "tracking clip" that can be tracked by infra-red cameras. Our "tracking clip" can be mounted on top of various surgical devices such as an endoscope. For this project, we used the infra-red vision-cameras by Image Guidance Technologies Inc. to track our tracking clip. The orientation of the projection axis and the position of the tip of the endoscope was calculated and extrapolated from the three known points (infra-red diodes) on the tracking clip holding the endoscope.

In order to register the imaging data-set with the patient's physical anatomy, we used six fiducial markers that were glued to the surface of the object during the CT and MR imaging. The object was then moved to the surgical site with the markers still attached. Registration was performed using standard orthogonal views (Axial, Sagital, Coronal) of the image data. For each marker, the technician positioned the tip of the infra-red diode-coded pointer (being tracked by infra-red cameras) on top of each marker, and positioned the computer cursor on top of the marker's imaging counterpart. Based on at least three sets of point pairs (not located in a plane), the registration system computed and forced the transformation matrix to register the patient's stereotactic space and the imaging space. At this point, since the patient and the volume image were registered, one could track the movement of an object around the patient, and the object's virtual counterpart was moved around the volume image with the same exact orientation. 


\subsection{Volumetric Data Overlay}

From the information obtained in section 4.3., the position of the endoscope's lens and its field of view, which is a volumetric conic section, can be obtained. In turn, these data allow the trajectory angles $\left(\theta_{x}, \theta_{y}, \theta_{z}\right)$ for the surgical endoscope to be calculated. At this point, if we rotate the 3-D data-set in reverse angle $\left(-\theta_{x},-\theta_{y},-\theta_{z}\right)$, the viewing projection will be the same as the endoscope's field of view. Thus, by multiplying the viewing projection matrix by the inverse matrix of the actual transformation matrix, the desired viewing angle can be obtained.

An imaginary cone, starting from the tip of the virtual endoscope and parallel to its trajectory axis represents our virtual endoscope's field of view (FOV). 2-D multi-layer modalities can be obtained by moving a vector along the surface of the cone (FOV). Using the parametric equation below, it is possible to calculate all the intersecting points between the cone and the volumetric data-set. Once the contours of the cone are defined, one can calculate all the voxels within the conic section:

$$
\begin{aligned}
& X=L \cdot \tan \theta \cos \alpha+x_{1} \\
& Y=L+y_{1} \\
& Z=L \cdot \tan \theta \sin \alpha+z_{1}
\end{aligned}
$$

Where $R=L \cdot \tan \theta$ is the radius in the base of the cone and $y_{1}, x_{1}, z_{1}$ are the coordinates of the origin of the cone, and $\theta$ is the angle between, the cone's axis and semi-axis. This formula represents a cone with its major axis along the $Y$ axis. To transfer this cone to the image's virtual space coordinates, we multiply $[X, Y, Z]$ by the object's orientation matrix. This orientation matrix is the inverse $(\rho)$ the orientation matrix obtained from the transformations of the actual image with respect to the cone. When we increment $\theta$ and $L$, the surface of the cone, as well as the intersection points between the cone and the image are all covered.

The rendering, after this alignment, is accomplished by reformatting a data-set along its common coordinate system to achieve a 2-D image overlay. Using this reformatting information and our 2-D Engine's oblique cutting plane capabilities, it is possible to make cuts - orthogonal to the cone's major axis - one layer at a time. These multimodalities can be observed as "raw" monochromatic images that are orthogonal and parallel to the tip of the trajectory device as it moves through the object.

This conic volume as a "see-through" projection in the 3-D data-set provides volumetric and multi-layer capabilities for the surgeon. With this system, the surgeon will have a choice of various image display mechanisms with our system. He/she can observe the actual endoscopic image and its virtual counterpart next to each other, or overlaid with each other with different blending parameters. Oblique 2-D multimodalities oriented to match the endoscope's trajectory can be displayed next to the 3-D images. With this technique, deep as well as superficial structures can be seen in context, considerably enhancing intra-operative guidance. 


\section{Results and Discussion}

The validation process followed in successive stages. Observing the volumetric data from the endoscope's perspective provided the means for testing the overall accuracy of our navigational system. The screen presentation of the endoscope's view of 3-D objects, were compared to the screen presentation of 3-D virtual objects at various depths. The images obtained from both modalities were compared to observe the differences in spacial occupancy of the anatomical structures. Each step mentioned above was also validated with variations in the field of view of the endoscope.

\subsection{Phantom Testing}

In order to test our system, we have used a phantom. This phantom (shown in figure 3: second from top, and right image) is a solid plastic mock-up of a skull, containing geometrical structures inside. In the same image the "tracking clip", which was designed and built by our group, is shown holding an endoscope. The geometrical structures consist of a rectangular cube, a cone, a cylinder and a sphere. The phantom also contains spatially fixed $2 \mathrm{~mm}$ holes, set $10 \mathrm{~mm}$ apart; and a $100 \times 100 \mathrm{~mm}$ grid pattern with $1 \mathrm{~mm}$ grids. There are 6 fiducial markers attached to the surface of the skull, the same as the ones being used during the actual stereotactic surgery. For MR imaging, the phantom is embedded in a water-sealed glass container and the container is filled with water. Once the container is emptied, it can be used for a CT imaging.

Using this phantom a series of feasibility experiments were conducted. The first involved the real time overlay of 3-D CT images with the phantom, using fiducial registration and an optical tracking system. Our initial goal was to simply obtain optimal registration (figure 3: two right images on the bottom). Our second goal was to maintain the spatial registration of the surgical tools (i.e., surgical pointer and endoscope) and the phantom, as the tool moved around and through the object (figure 3: second and third from top, and left image). The third involved perspective volumetric image overlay with the actual endoscopic trajectory views (figure 3: two top images). Subsequent sectioning of the image allowed for a precise quantization of the system's targeting accuracy and image/anatomy overlap at different depths.

\subsection{Overall Results}

Figure 2, shows the overall performance of our system. There have been 100 random cases studied. In each case, the Least-Square-Mean (LSM) error based on pixeloffsets were measured, and plotted versus the depth of observation, and the field of view of the endoscope. On the top-left of figure 2, the Least-Square-Mean vs. depth is represented on a 3D Plot. It is illustrated that, as the field of view increases, the errors tend to increase on the edge of the reconstructed field; and the deeper the measurements from the tip of the endoscope, the more error is obtained. The best reconstruction results were obtained using the following parameters: the depths of up to $25 \mathrm{~mm}$ with the observation toward the center of the endoscope's viewpoint. Using these parameters the worst scenario yields less than $1.5 \mathrm{~mm}$ offset. Over all, we have demonstrated the precision of our reconstruction to be around $3.0 \mathrm{~mm}$ in the worst case (with FOV equals to 120 degrees and at depths of $50 \mathrm{~mm}$ ). In real surgical scenario these errors might increase, due to the intraoperative deformation of tissue. 


\subsection{Sources of Error}

Overall the accuracy of the system is directly dependent on the precision with which the registration is conducted. In addition, the results are also dependent on the amount of lens distortion, its focal length and field of view. Moreover, for any given video image, there is a sampling error that can be estimated as a fraction of the largest extent of the imaged object. For example, the highest resolution image that can be made by a standard NTSC video image is $640 \times 480$ pixels. This would give the precision of $1 \mathrm{~mm} /$ pixel for an object with an extent on the window of $512 \times 512$ pixels. Thus, the precision of the instrument is also related to the distance of the camera from the object, the film resolution and the lens quality.

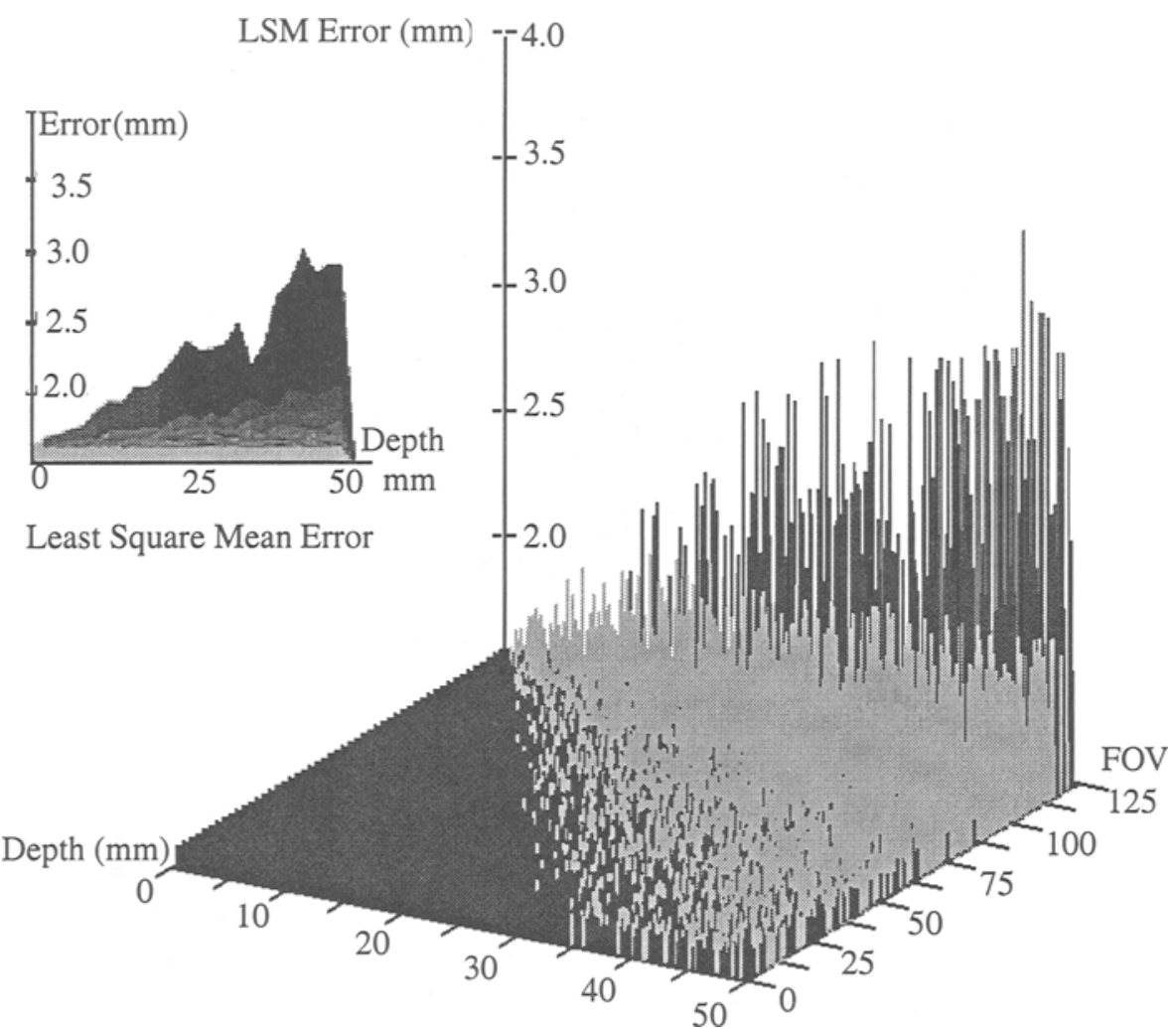

Figure 2: Results of 100 random cases of endoscopic's reconstruction study with variables field of views at different depths.

A lot more testing and statistical analysis are needed to validate the accuracy of our system. Next, we will test our system on deformable phantoms and also cadavers with target lesions inserted in them. Finally, we will test our system in a real surgical scenario, where a volumetric virtual endoscopic updates will be compared with the video images obtained from an endoscope, looking at the patient's physical anatomy. 

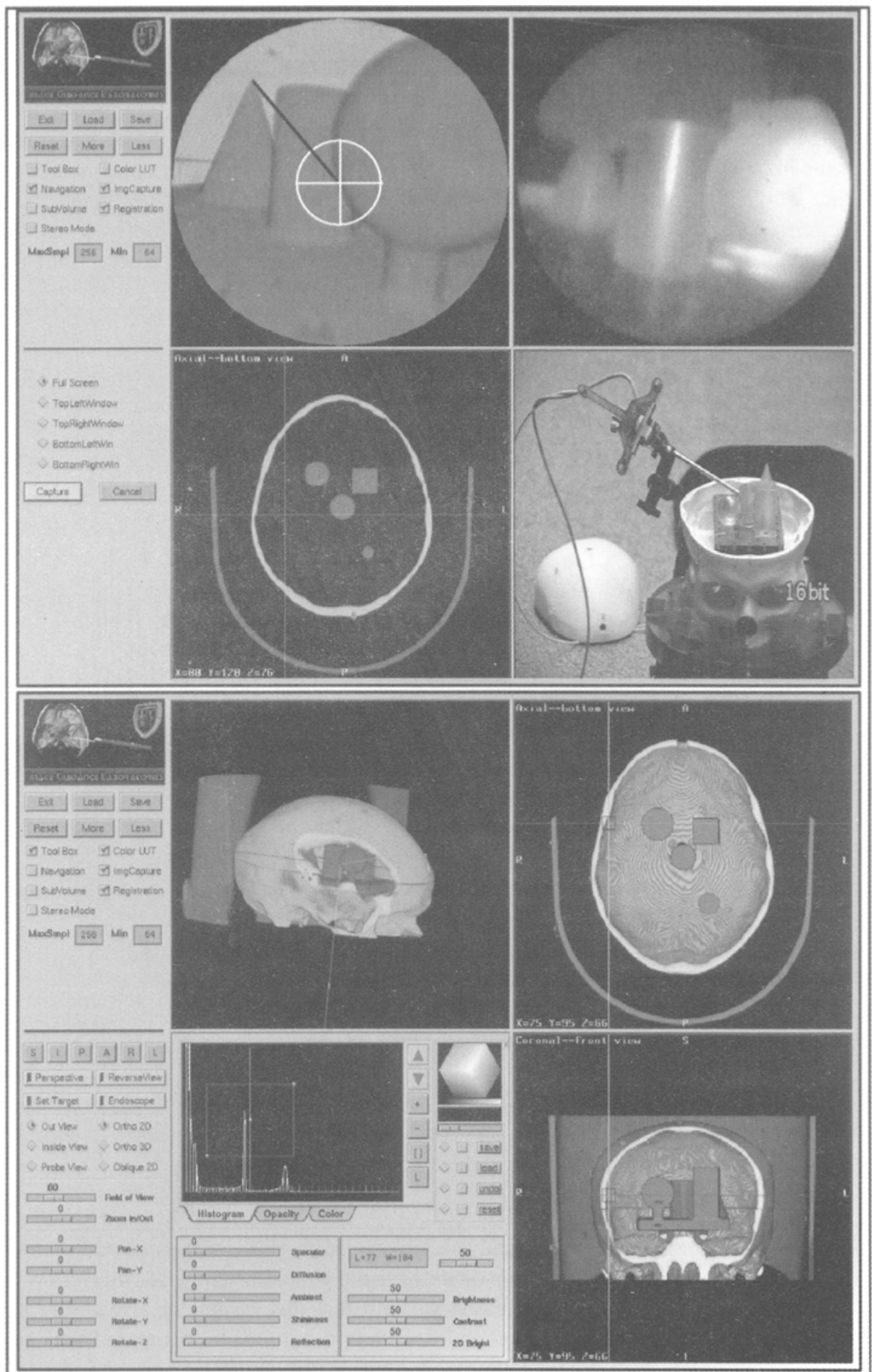

Figure 3: Various viewing options using "Genesis2000" surgical navigation software. 


\section{Future Work}

We are currently working on a multi-step lens calibration to compensate for lens distortion. The first step will be to compensate for the lens curvature. The second step will be the initial calibration stage to automatically incorporate the lens' field of view and focal length into our system. The last step will be an automated matching of the stereoscopic endoscopic images, and the stereoscopic 3-D reconstructed images based on a real-time surface matching of the localized area, as seen through the endoscope. Another project currently under investigation in our lab, is the development of a system which would utilize intra-operative ultrasonic images as sources of the patient data, in order to compensate for intraoperative tissue deformations. Volumetric pre-operative CT or MR imagery can then be spatially deformed (warped) to match the intra-operatively acquired 3-D US images. Such images can then be fused with images from the surgical endoscope or microscope.

\section{References}

1. TM. Peters, A. Olivier. "CT-aided stereotaxy for depth electrode implantation and biobsy," Canadian Journal of Neurological Sciences, 10:166-169, 1983.

2. P. Gildenberg, HH. Kaufman, KSM. Murthy, "Calculation of stereotactic coordinates from computed tomography scan," Neurosurgery, 10:580-586, 1982.

3. P. Kelly, B. Ball, S. Goerss, "Results of computer tomography-based stereotactic resection of metastatic intracranial tumors," Neurosurgery 22:7-17, 1988.

4. RJ. Maciunas, RL. Galloway, JW. Latimer. "The Application Accuracy of Stereotactic Frames Neurosurgery," 35(4):682-695, 1994.

5. B. Guthrie and JR. Adler. Frameless Stereotaxy: Computer Interactive Neurosurgery. Perspectives in Neurological Surgery. 2(1):1-22, 1991.

6. WB. Legget, MM. Greenberg, WEJ. Gannon, "The Viewing wand: a new system for three-dimensional CT correlated intraoperative localization," Current Surgery, 1991, 48:674-678.

7. P.F. Hemler, T. Koumrian, J.R. Adler, and B. Guthrie," A Three Dimensional Guidance System for Frameless Stereotactic Neurosurgery," In Proc. of the Fifth Annual IEEE Symposium on Computer-Based Medical Systems, pages 309-314, Durham, North Carolina, 6/92.

8. JW. Trobaugh, WD. Richard, KR. Smith, RD. Bucholz, "Frameless stereotactic ultrasonography: method and applications," Compute Med. Imaging Graph 1994 Jul;18(4):235-246.

9. KR. Smith, KJ. Frank, and RD. Bucholtz. "The Neurostation - A Highly Accurate, Minimally Invasive Solution to Frameless Stereotactic Neurosurgery," Computerized Medical Imaging and Graphics, 18(4):247-256, 1994.

10. P. Cinquin, E. Bainville, C. Barbe, et., al,."Computer Assisted Medical Interventions," IEEE Engineering in Medicine and Biology, pages 254-263, 1995.

11. S. Lavallee, J. Troccaz, P. Sautot, B. Mazier, P. Cinquin, and P. Merloz, "Computer- Assisted Spinal Surgery Using Anatomy-Based Registration. In ComputerIntegrated Surgery,' R.H. Taylor et al. (eds), pp 425-449, MIT Press, 1995.

12. LP. Nolte, H. Visarius, E. Arm, F. Langlotz, O. Schwarzenbach, L. Zamorano, “Computer Aided Fixation of Spinal Implants," Jour of IGS, 1:65-73, 1995. 
13. HF. Reinhardt. "Neuronavigation: A Ten-Year Review. In Computer-Integrated Surgery," RH. Taylor et al. (eds), pages 329-341, MIT Press, 1995.

14. RD. Bucholtz, DD. Yeh, J. Trobaugh, et., al. "The Correction of Stereotactic Inaccuracy Caused by Brain Shift Using an Intraoperative Ultrasound Device," in Proc. CVRMed-MRCAS'97, pages 459-466, Grenoble, France, 1997.

15. R. Kikinis, 1. Gleason, W. Lorensen, W Wells, WE Grimson, T Lozano-Perez, G. Ettinger, S. White, F. Jolesz, “ Image Guided Techniques for Neurosurgery," In Proc. VBC 94, pp. 537-540, Rochester, 1994.

16. R. Shahidi, "Applications of Virtual Reality in Stereotactic Procedures: Volumetric Image Navigation via Surgical Microscope" Ph.D. Thesis, Rutgers University, Submitted 1994.

17. W.E.L. Grimson, T. Lozano-Perez, W. M. Wells III, G. J. Ettinger, S. J. White, and R. Kikinis. "An Automatic Registration Method for Frameless Stereotaxis, Image Guided Surgery, and Enhanced reality Visualization," in Proc. IEEE Compt Vis and Patt. Recog., pp. 430-436, 1994.

18. ACF Colchester, J Zhao, CHenri, RL Evans, P Roberts, N. Maitland, DJ Hawkes, DLG Hill, AJ Strong, DG. Thomas, MJ Gleeson, TCS Cox," In Proc. VBC 94, pp. 541-551, Rochester, 1994.

19. R. Shahidi, R. Mezrich, D. Silver, "Proposed Simulation of Volumetric Image Navigation Using a Surgical Microscope," Jour Img Guid Surg, 1:249-265, 1995.

20. A.C.F. Colchester et al. Development and Preliminary Evaluation of VISLAN, a Surgical Planning and Guidance System Using Intraoperative Video Imaging. Med Image Analysis, 1(1):73-90, 1996.

21. K. Darabi, KD. Resch, J. Weinert, U. Jendrysiak, A. Perneczky, " Real and Simulated Endoscopy of Neurological Approaches in an Anatomical Model", In Proc., CVRMed-MRCAS, pp. 323-326, Grenoble, France, 1997.

22. Robert A. Drebin, Loren Carpenter, and Pat Hanrahan. "Volume rendering. Computer Graphics" (ACM SIGGRAPH Proceedings), 22(4):65-74, 1988.

23. M. Levoy, "Display of Surfaces from Volume Data", IEEE Computer graphics \& Applications 0272-1716/88/0500-0029., 1988.

24. M. Levoy, "A Hybrid Ray Tracer for Rendering Polygon and Volume Data. IEEE Computer graphics \& Applications 0272-1716/90/0300-0033., 1990.

25. M. Levoy, "Efficient Ray Tracing of Volume Data", ACM Trans. Graph., 9(3): $245261,1990$.

26. B. Cabral, N. Cam, J. Foran. Accelerated Volume Rendering and Tomographic Reconstruction using Texture Mapping Hardware. In Proceedings of the 1994 Symposium on Volume Visualization, pages 91-98, 1994.

27. P. Lacroute and M. Levoy, "Fast Volume Rendering Using a Shear-Warp Factorization of the Viewing Transformation," In Proc. of SIGGRAPH '94, Orlando, FL, pages 451-458, 1994.

28. Kortokov E., Henriksen K., Kories R., Stereo Ranging with Verging Cameras, IEEE PAMI, Vol. 12, NO. 12 Dec. 1990.

29. Alvertos N., Brzakovic D., Gonzalez R C., Camera Geometries for Image Matching in 3D Machine Vision: IEEE on Pattern Analysis and Machine Intelligence (PAMI), Vol 11, NO. 9. 9/1989. 